\title{
RSV vs. rhinovirus bronchiolitis: difference in nasal airway microRNA profiles and NFKB signaling
}

Kohei Hasegawa' ${ }^{1}$, Marcos Pérez-Losada ${ }^{2}$, Claire E. Hoptay ${ }^{3}$, Samuel Epstein ${ }^{3}$, Jonathan M. Mansbach ${ }^{4}$, Stephen J. Teach ${ }^{5}$, Pedro A. Piedra ${ }^{6}$, Carlos A. Camargo $\mathrm{Jr}^{1}$ and Robert J. Freishtat ${ }^{7}$

BACKGROUND: Although rhinovirus infection is associated with increased risks of acute and chronic respiratory outcomes during childhood compared with respiratory syncytial virus (RSV), the underlying mechanisms remain unclear. We aimed to determine the differences in nasal airway microRNA profiles and their downstream effects between infants with rhinovirus and RSV bronchiolitis.

METHODS: As part of a multicenter cohort study of infants hospitalized for bronchiolitis, we examined nasal samples obtained from 16 infants with rhinovirus and 16 infants with RSV. We tested nasal airway samples using microarrays to profile global microRNA expression and determine the predicted regulation of targeted transcripts. We also measured gene expression and cytokines for NFkB pathway components.

RESULTS: Between the virus groups, 386 microRNAs were differentially expressed (false discovery rate $(F D R)<0.05$ ). In infants with rhinovirus, the NFKB pathway was highly ranked as a predicted target for these differentially expressed microRNAs compared with RSV. Pathway analysis using measured mRNA expression data validated that rhinovirus infection had upregulation of NFKB family (RelA and NFKB2) and downregulation of inhibitor $\mathrm{KB}$ family. Infants with rhinovirus had higher levels of NFkB-induced type-2 cytokines (IL-10 and IL-13; FDR<0.01).

CONCLUSION: In infants with bronchiolitis, rhinovirus and RSV infections had different nasal airway microRNA profiles associated with NFKB signaling.

B ronchiolitis is an important public health problem in the United States (1). Indeed, bronchiolitis is the leading cause of hospitalizations for US infants, with $\sim 130,000$ hospitalizations each year (1). In addition to this acute morbidity, bronchiolitis has associated chronic morbidity; $30-40 \%$ of infants hospitalized with bronchiolitis develop childhood asthma (2). Analyses of the two major causative viruses (rhinovirus and respiratory syncytial virus (RSV)) suggest that rhinovirus infection is associated with distinct host immune response profiles (3), and with different risks of acute (e.g., bronchiolitis severity) and chronic (e.g., incident asthma) respiratory outcomes during childhood when compared to RSV infection (2). Although these studies suggest that respiratory virus infection and airway immune response modulation are associated with respiratory outcomes in infants with bronchiolitis, the underlying mechanisms of these links remain unclear (4).

The recent discovery of interactions between innate and adaptive immune responses in the airway is beginning to reveal potential mechanisms-e.g., viral-induced programming of airway immune response via epigenetic changes involving microRNAs (5). MicroRNAs comprise a large family of highly conserved, non-coding, short, singlestranded RNAs that regulate $\sim 60 \%$ of protein-encoding genes via promoting mRNA degradation or inhibiting translation (6). Although the literature remain sparse, we recently demonstrated that rhinovirus infection elicits expression of specific microRNAs (e.g., miR-155) in the nasal airway of young children (7). In addition, studies have reported that rhinovirus infection not only activates NFKB signaling pathway (8-11), but also subsequently induces airway hyperreactivity (12). No prior study, however, has compared the microRNA profiles and their downstream signaling pathways between the two major respiratory virusesrhinovirus and RSV-in children.

To address this knowledge gap, we examined infants hospitalized for bronchiolitis enrolled into a multicenter cohort study to determine the differences in the nasal airway microRNA profiles and their downstream effects (gene and cytokine expression) between rhinovirus and RSV infections. Specifically, we hypothesized that, compared with RSV infections, rhinovirus infections would be associated with

\footnotetext{
'Department of Emergency Medicine, Massachusetts General Hospital, Harvard Medical School, Boston, Massachusetts; ${ }^{2}$ Department of Integrative Systems Biology, George Washington University School of Medicine and Health Sciences, Washington, DC; ${ }^{3}$ Center for Genetic Medicine Research, Children's National Health System, Washington, DC; ${ }^{4}$ Department of Medicine, Boston Children's Hospital, Boston, Massachusetts; ${ }^{5}$ Department of Pediatrics, George Washington University School of Medicine and Health Sciences and the Division of Emergency Medicine, Children's National Health System, Washington, DC; ${ }^{6}$ Department of Molecular Virology and Microbiology and Pediatrics, Baylor College of Medicine, Houston, Texas; ${ }^{7}$ Division of Emergency Medicine, Children's National Health System, Washington, DC. Correspondence:

Kohei Hasegawa (khasegawa1@partners.org)

Received 23 May 2017; accepted 25 November 2017; advance online publication 17 January 2018. doi:10.1038/pr.2017.309
} 
distinctive microRNA signatures that upregulate $\mathrm{NF \kappa B}$ signaling in the nasal airway of infants with bronchiolitis.

\section{METHODS}

Study Design, Setting, and Participants

We analyzed data from an ongoing multicenter prospective cohort study of infants (age $<1$ year) with severe bronchiolitis-the 35th Multicenter Airway Research Collaboration (MARC-35) (13-17). MARC-35 is coordinated by the Emergency Medicine Network (EMNet), a collaboration of 245 participating hospitals. Using a standardized protocol, site investigators at 17 sites across 14 US states enrolled 1,016 infants hospitalized with an attending physician's diagnosis of bronchiolitis during three consecutive bronchiolitis seasons from 1 November 2011 to 30 April 2014. Bronchiolitis was defined by the American Academy of Pediatrics guidelines: acute respiratory illness with some combination of rhinitis, cough, tachypnea, wheezing, crackles, and retractions (18). We excluded infants with known heart-lung disease, immunodeficiency, immunosuppression, or gestational age $<32$ weeks, those who were transferred to a participating hospital $>24 \mathrm{~h}$ after the original hospitalization, or those who were consented $>24 \mathrm{~h}$ after hospitalization. All patients were treated at the discretion of the treating physicians. The institutional review board at each of the participating hospitals approved the study. Written informed consent was obtained from the parent or guardian.

In the present study, we randomly selected 16 infants with sole rhinovirus infection and 16 infants with sole RSV infection (i.e., no co-infecting viruses) from the MARC-35 cohort, and investigated global microRNA and mRNA expression as well as cytokine levels in the nasal airway.

\section{Data Collection}

At the index hospitalization, site investigators conducted a structured interview that assessed patients' demographic characteristics, medical and family history, and details of the acute illness. Emergency department and hospital chart reviews provided further clinical data, such as vital signs, physical examination, medical management, and disposition. Review of medical records was performed, after successful completion of training (lecture, practice charts), by board-certified physicians (e.g., from pediatric pulmonary, allergy/ immunology). All data were reviewed at the EMNet Coordinating Center at Massachusetts General Hospital (Boston, MA), and site investigators were queried about missing data and discrepancies identified by manual data checks.

On the basis of evidence that nasal airway inflammatory response is indicative of that in the lower respiratory tract (19-21), we investigated nasal airway specimens. Trained investigators collected nasal swabs from the anterior nares, using a standardized protocol (22), within $24 \mathrm{~h}$ of hospitalization. Both nares were swabbed with a single nylon, pediatric FLOQSwab (Copan, Brescia, Italy). Nasal airway specimens were tested for (1) respiratory viruses, including rhinovirus and RSV, using real-time polymerase chain reaction (PCR) assays, (2) microRNA expression, and (3) mRNA expression, as well as (4) cytokine levels.

\section{RNA Extraction and MicroRNA Microarray}

Total RNA from the nasal airway specimens was isolated using a Norgen RNA/DNA Purification Kit (Norgen Biotek, Thorold, ON, Canada) and amplified using a Seramir Exosome RNA Amplification Kit (System Biosciences, Palo Alto, CA). MicroRNA quality was determined by Nanodrop1000 (Thermo Scientific, Wilmington, DE) with absorbance ratios for UV $260 / 280 \geq 2.0$ and $260 / 230$ between 1.8 and 2.2. Those samples meeting quality control criteria were hybridized to Affymetrix GeneChip microRNA 4.0 arrays (Affymetrix, Santa Clara, CA). Resulting data were analyzed in Expression Console using RMA+DMBG (Affymetrix), then exported to Partek Genomics Suite (Partek, St. Louis, MO) for the analyses.

\section{mRNA Measurement}

Although NFKB measurements are typically performed in cell-based systems with reporter constructs (23), the MARC-35 nasal swab specimens were not cell-based, and thus required a different approach. For these specimens, we measured mRNAs and cytokines not only for the components of NFKB signaling pathway, but also for inflammatory mediators reliably induced by $\mathrm{NF \kappa B}$ as an indirect measure of NFkB activity (24-26).

To test the changes in NFKB signaling-related mRNAs as a result of nasal airway microRNA differences, we first prepared cDNA from the RNA extracted from the nasal airway specimens and preamplified the NFKB-specific genes by using the $\mathrm{RT}^{2}$ PreAMP cDNA Synthesis Kit (Qiagen, Valencia, CA). The preamplified cDNA was input into the $\mathrm{RT}^{2}$ ProfilerTM PCR Array for Human NFאB Signaling Pathway (Qiagen), a qRT-PCR array that allows for the simultaneous mRNA profiling of 84 genes related to NFKB signaling, in addition to housekeeping genes. An array for each case was run on an ABI 7900HT Fast Real-Time PCR System (Applied BioSystems, Carlsbad, $\mathrm{CA}$ ) and data were analyzed using the $\mathrm{RT}^{2}$ Profiler PCR Array Data Analysis software, version 3.5 (Qiagen).

\section{Cytokine Measurement}

To test the differences in $10 \mathrm{NF \kappa B}$ signaling-related cytokine expressions (GM-CSF, IFN $\gamma$, IL-1 $\beta$, IL-2, IL-6, IL-7, IL-8, IL-10, IL-13, and TNF $\alpha$ ) between the two virus groups, we tested nasal airway specimen supernatants using the Milliplex MAP Human High Sensitivity T-cell Panel Premixed magnetic bead-based assay (EMD Millipore, Billerica, MA) on the MAGPIX System (EMD Millipore). Data were analyzed using the Milliplex Analyst 5.1 software (EMD Millipore).

\section{Statistical Analyses}

All nasal airway samples passed quality control tests and were used for the microRNA analysis. Processed microRNA data were normalized using generalized log non-linear transformations. Differences in the microRNA expression profiles between rhinovirus and RSV groups were examined using principal coordinates analysis (PCoA) with the Bray-Curtis distance, as well as unsupervised hierarchical clustering using Spearman's rank correlation similarity and the Ward's algorithm. Benjamini-Hochberg false discovery rate (FDR) multiple test correction was applied. Both analyses were carried out in RStudio (RStudio, Boston, MA).

To identify microRNAs and mRNAs that are differentially expressed between the two virus groups, we performed analysis of variance in Partek Genomics Suite version 6.6 (Partek, St. Louis, $\mathrm{MO}$ ). Next, to investigate the cumulative effects of microRNAs on the gene expression regulation among infants with rhinovirus infection (in comparison to those with RSV), we uploaded the differentially expressed microRNAs into Ingenuity Pathway Analysis (IPA). We carried all microRNAs that had a $P<0.10$ and a fold difference of $>4.0$ cutoff into the IPA analysis. Targets of microRNAs were determined using the IPA microRNA Target Filter, which identifies experimentally validated microRNA-mRNA interactions from TarBase, miRecords, and biomedical literature, and predicted microRNA-mRNA interactions from TargetScan. We used a conservative filter, using only experimentally validated and highly conserved predicted mRNA targets for each microRNA. We used these mRNA targets in the Core Pathway Analyses, which identified relationships among the mRNAs in our data set. Canonical pathways, novel networks, and common upstream regulators were then queried for overlap with our differentially expressed microRNA gene target list. Last, we compared the difference in pathway enrichment between the virus groups using the Benjamini-Hochberg FDR multiple test correction.

In addition to the IPA analysis, we also used miRTarVis (27) to visualize the microRNA-target mRNA expression interaction network. This bioinformatic approach integrates microRNA and mRNA expression profiles, and predicts targets of microRNA by adopting Bayesian inference, MINE analyses, conventional correlation, and 


\section{Articles | Hasegawa et al.}

mutual information analyses. Lastly, to examine the downstream effect of microRNAs, we used the Mann-Whitney $U$-test to determine differences in the NFKB signaling-related cytokine levels between the virus groups.

\section{RESULTS}

\section{Study Population}

As a part of an ongoing multicenter prospective cohort study, we examined nasal airway samples from 16 infants with rhinovirus bronchiolitis and 16 infants with RSV bronchiolitis. In the current investigation, the analytic and nonanalytic cohorts had no significant differences in most patient characteristics $(P>0.05$; Supplementary Table S1 online), except the analytic cohort, which had a relatively higher proportion of hypoxemia upon presentation $(P=0.02)$. Of 32 infants in the analytic cohort, the median age was 3 months (interquartile range, 2-7 months), 69\% were male, and 50\% were non-Hispanic white. Between the virus groups, there were no significant differences in the baseline patient characteristics, clinical presentation, or hospitalization course (all $P>0.10$; Table 1).

\section{Nasal Airway MicroRNA Expression Profile Differs by Infecting} Virus

The analysis of global microRNA expression identified 2,758 microRNAs in the nasal airway of infants hospitalized for bronchiolitis. Of these microRNAs, 386 were differentially expressed between the two virus groups $(P<0.05$ with FDR correction). In the PCoA plot (Figure 1), the microRNA expression profiles almost completely separated infants with rhinovirus bronchiolitis from those with RSV bronchiolitis. Similarly, the unsupervised hierarchical clustering segregated most patients from each viral group (Figure 2).

Infants with Rhinovirus Bronchiolitis had Specific Nasal Airway MicroRNA Signature that Enhances NFkB Signaling Pathway

To investigate the cumulative effects of virus-specific microRNA profiles on gene expression, the differentially expressed microRNAs were used for IPA analysis. As hypothesized $a$ priori, the NFKB pathway was highly ranked as a predicted target for these differentially expressed microRNAs $(P<0.0001$ with FDR correction; Supplementary Table S2 online). Of 180 genes in the NFKB pathway, 137 genes were predicted to be targeted by these microRNAs. As shown in Figure 3a, infants with rhinovirus bronchiolitis had predicted downregulation of the inhibitor $\kappa \mathrm{B}$ (I $\kappa \mathrm{B}$ ) family, the major inhibitory proteins of the NFKB signaling pathway, when compared with infants with RSV bronchiolitis. To validate our inference of the microRNAs' cumulative effects on the NFkB signaling pathway, we also measured the global expression of 84 genes related to $\mathrm{NF \kappa B}$ signaling in the nasal airway. Consistent with the predicted regulation of NFkB signaling pathway, the pathway analysis using the measured mRNA expression data (Figure 3b) also demonstrated that infants with rhinovirus bronchiolitis had downregulation of $\mathrm{I} \kappa \mathrm{B}$. In contrast, these infants had upregulation of RelA (p65) and NFkB2 (p100/p52)proteins in the NFאB family.

Likewise, the integrated analysis of microRNA and mRNA expression with the use of miRTarVis demonstrated consistent findings (Figure 4a,b). For example, infants with rhinovirus bronchiolitis had upregulation of multiple microRNAs that downregulate expression of $N F K B I B$ (the gene encoding IкB- $\beta$ )-e.g., hsa-miR-149-3p (4.2-fold increase; $P<0.001)$, hsa-miR-197-3p $\quad(5.5$-fold increase; $P<0.001)$, hsa-miR-197-5p (4.3-fold increase; $P<0.001)$, and hsa-miR-296-3p (5.3-fold increase; $P<0.001)$-when compared to infants with RSV infection (Figure 4a and Supplementary Table S3 online). Similarly, infants with rhinovirus had upregulation of microRNAs targeting another IкB family gene, NFKBIE-e.g., hsa-miR-149-3p (4.2-fold increase; $P<0.001)$ and hsa-miR-504-3p (4.1-fold increase; $P<0.001)$. Last, in these patients, hsa-miR-155-5p expression was also upregulated (4.3-fold increase; $P<0.001)$. By contrast, infants with rhinovirus infection had downregulation of many microRNAs targeting RELA, the gene encoding RelA, when compared to infants with RSV infection (Figure 4b and Supplementary Table S3 online). Likewise, these infants had downregulation of multiple microRNAs targeting FOS, expression of which is known to be upregulated by the $\mathrm{NF \kappa B}$ pathway in conjunction with the extracellular signal-regulated kinase pathway $(28,29)$.

We also measured ten NFKB-regulated cytokines in the nasal airway. Infants with rhinovirus bronchiolitis had higher levels of IL-10 and IL-13 compared with those infants with RSV bronchiolitis (both $P<0.05$ with FDR correction; Supplementary Table S4 online). The production of IL-10 and IL-13 is known to be induced by the NFKB signaling pathway $(30,31)$.

\section{DISCUSSION}

In this analysis of the data from an ongoing multicenter cohort of infants with bronchiolitis, we found that the nasal airway microRNA profiles differ between infants infected with rhinovirus and RSV. We also found that infants with rhinovirus infection had an altered microRNA profile that is predicted to greatly enhance the $\mathrm{NF \kappa B}$ signaling pathway when compared to infants with RSV infection. This finding was mirrored by the observations that the rhinovirus-related microRNA signature is associated with measured downregulation of $\mathrm{I} \kappa \mathrm{B}$ family genes and upregulation of $\mathrm{NF \kappa B}$ genes. Consistent with the literature (32), infants with rhinovirus infection had higher levels of NFKB-induced type- 2 cytokines (IL-10 and IL-13) in comparison to those with RSV infection. To the best of our knowledge, this is the first investigation to have examined the difference in microRNA signatures between rhinovirus and RSV and its downstream effects in the setting of severe viral respiratory infection.

The literature indicates that microRNAs help maintain the normal development of the airways and lung in infancy, and throughout childhood help fine-tune airway inflammatory 
Table 1. Characteristics and clinical presentation of infants hospitalized for bronchiolitis by associated viral infection

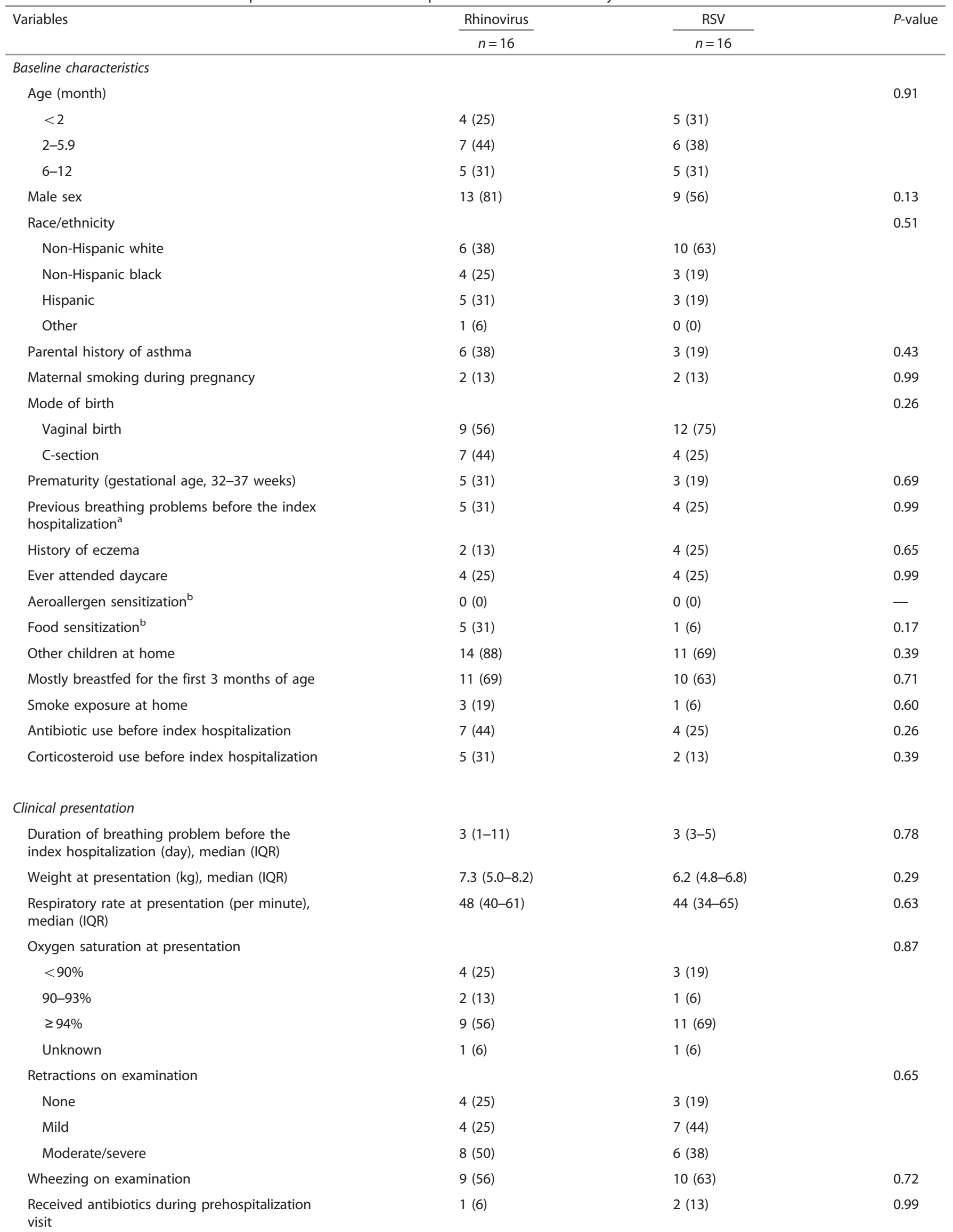




\section{Articles | Hasegawa et al.}

Table 1 Continued

\begin{tabular}{llcc}
\hline Variables & Rhinovirus & RSV & \multicolumn{1}{c}{ P-value } \\
\cline { 3 - 4 } & $n=16$ & 16 & $1(6)$ \\
\hline $\begin{array}{l}\text { Received corticosteroids during pre- } \\
\text { hospitalization visit }\end{array}$ & $4(25)$ & \\
Hospitalization course & & \\
Intensive care use & & $3(19)$ \\
Hospital length-of-stay $\geq 3$ days & $3(19)$ & $9(56)$ & 0.33 \\
Hospital length-of-stay (day), median (IQR) & $7(44)$ & $3(2-3)$ & 0.48 \\
\hline
\end{tabular}

$\mathrm{IQR}$, interquartile range; $\mathrm{n} / \mathrm{a}$, not applicable; RSV, respiratory syncytial virus.

Data are number (\%) of infants unless otherwise indicated. Patient characteristics, clinical presentation, and hospital course were compared by virus using $X^{2}$-test, Fisher's exact test, or Wilcoxon rank-sum test, as appropriate.

aDefined as an infant having cough that wakes him/her at night and/or causes emesis, or when the child has wheezing or shortness of breath without cough.

${ }^{b}$ Defined as admission to intensive care unit and/or use of mechanical ventilation (continuous positive airway pressure ventilation and/or intubation).

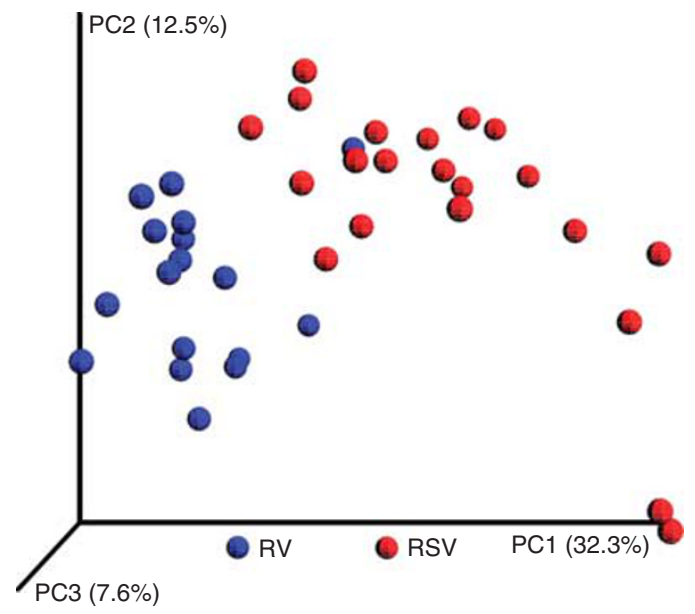

Figure 1. Principal coordinates analysis ( $\mathrm{PCOA})$ plot comparing nasal airway microRNA profiles in infants with rhinovirus bronchiolitis and those with RSV bronchiolitis. To show the differences in nasal airway microRNA profiles among infants with bronchiolitis, PCoA plot based on the Bray-Curtis distance was generated. Each dot represents the overall microRNA expression in each infant. The distance between infants indicates their dissimilarity. The PCOA revealed that infants cluster together according to their viral etiology. In addition to 16 samples with rhinovirus and 16 with RSV, 6 technical replicates were also included in the analysis. RSV, respiratory syncytial virus; RV, rhinovirus.

processes, including respiratory infections and asthma (4). Indeed, emerging evidence, mostly from in vitro investigations, has shown that RSV infection, through altering microRNA expression in airway epithelium, modulates immune responses in the airway $(33,34)$. Although the research on rhinovirus infection-related perturbations in microRNA expression is sparse, we recently examined the microRNA expression in the nasal airway of 10 young children (aged $<3$ years) with PCR-confirmed rhinovirus infection, and found that rhinovirus infection induces miR-155 when compared with 10 healthy children (7). In the current study, we also demonstrate upregulation of this microRNA among infants with rhinovirus infection in

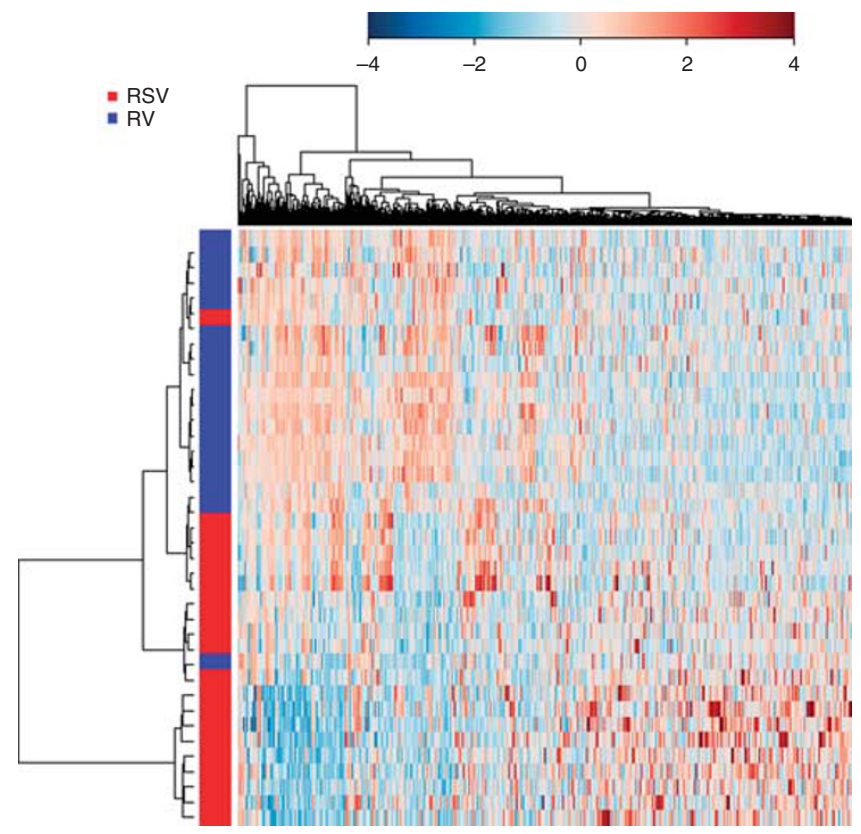

Figure 2. Unsupervised hierarchical clustering of the expression of identified microRNAs in nasal airway of infants with bronchiolitis. The heatmap of 2,758 microRNAs that are identified in the nasal airway was generated using the Spearman's rank correlation similarity and the Ward's clustering algorithm. The microRNA expression profiles almost completely separated infants with rhinovirus bronchiolitis from those with RSV bronchiolitis. The color bar indicates the standardized expression of each microRNA to a mean of 0 . Upregulated microRNAs have positive values and are displayed as red. Downregulated microRNAs have negative values and are displayed as blue. The differences in microRNA expression between rhinovirus and RSV are summarized in Supplementary Table S2 online. RSV, respiratory syncytial virus; RV, rhinovirus.

comparison to those with RSV. Studies have shown that miR-155 has critical roles in type- 2 pro-asthmatic responses, including Th2 priming (35), type-2 immune polarization (36), modulation of responses to IL-13 (37), and allergic airway inflammation (38). Our study corroborates previous reports linking respiratory virus infection, microRNA-related 

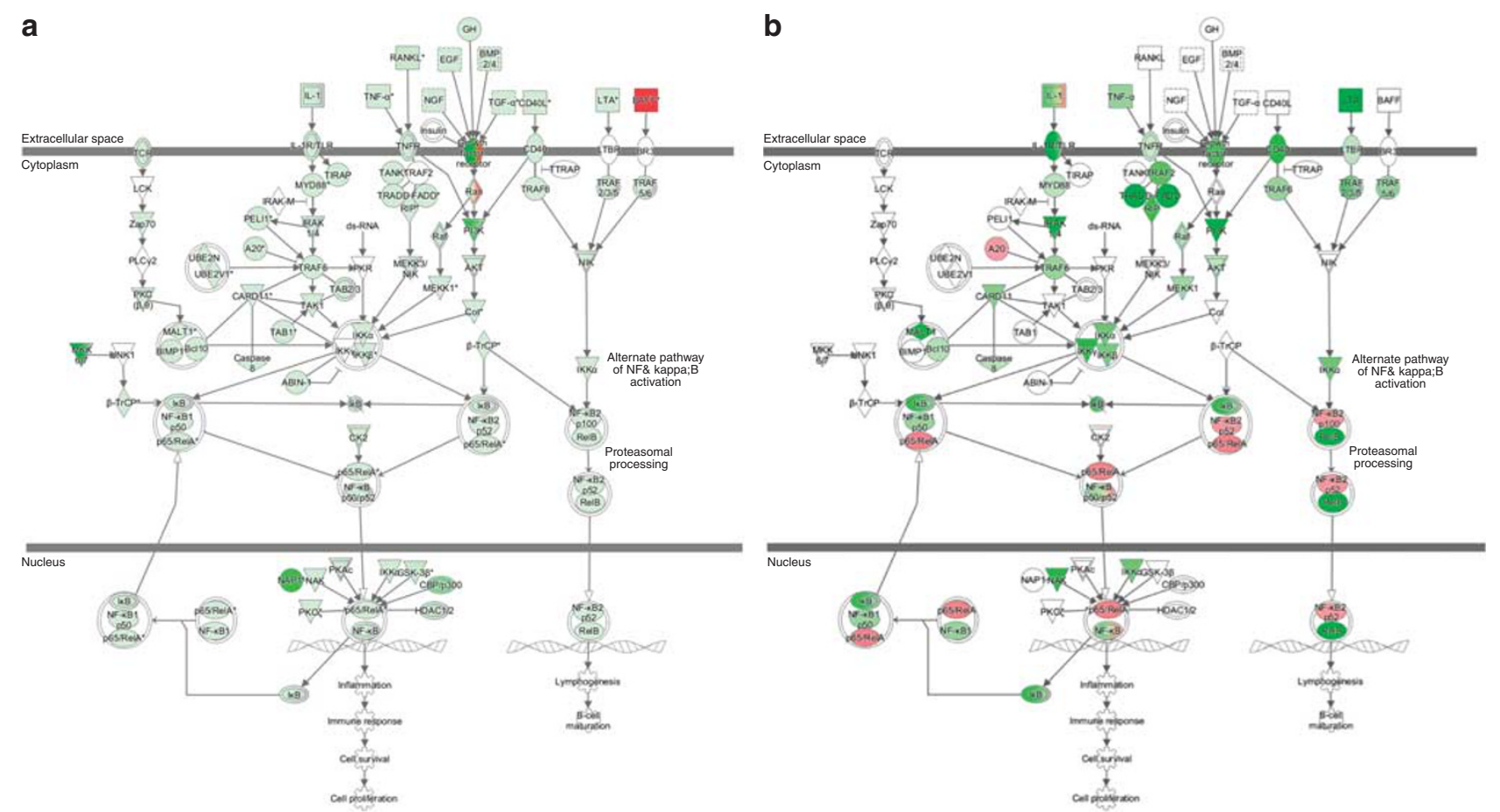

Figure 3. NFKB signaling pathway in the nasal airway comparing infants with rhinovirus bronchiolitis to those with RSV bronchiolitis. (a) Predicted up- and downregulation of target transcripts in the NFKB signaling pathway. The canonical pathway for NFKB signaling was highly ranked as a target for the microRNAs in infants with rhinovirus bronchiolitis compared to those with RSV bronchiolitis. The green color indicates predicted downregulation of transcripts targeted by differentially expressed microRNAs in the nasal airway of infants with rhinovirus bronchiolitis compared to those in infants with RSV; the red color indicates predicted upregulation. Genes are targeted by multiple microRNAs. (b) Measured up- and downregulation of target transcripts in the NFKB signaling pathway via RT-PCR.

immune modulation, and asthma. Our data extend these prior studies by demonstrating that rhinovirus infection-related microRNA signatures enhance the NFkB signaling pathway in infants during an important period of lung development (median age of 3 months).

The clinically relevant implication that rhinovirus infectionrelated perturbation of microRNA expression and activated NFKB signaling pathway may impact acute (e.g., bronchiolitis severity) and chronic (e.g., development of childhood asthma) bronchiolitis morbidity warrants further clarification. Multiple studies have reported that rhinovirus infection not only activates the NFKB signaling pathway (8-11), but also subsequently induces airway hyperresponsiveness (12). In addition, activation of the NFKB signaling pathway within the airway epithelium has been implicated in asthma pathobiology (e.g., allergic airway inflammation, airway hyperresponsiveness, and fibrotic airway remodeling) in animal models $(39,40)$. In addition, studies of adults with asthma have demonstrated that enhanced $\mathrm{NFKB}$ signaling, normally transient due to concurrent induction of the inhibitor $\kappa \mathrm{B}$, is persistent with resulting pathologic changes in immune cell cytokine/chemokine secretion $(41,42)$. Furthermore, Panganiban et al. (43), by profiling the microRNA expression in 35 adults with asthma, found that these patients had specific microRNA signatures (e.g., upregulation of miR-155) and that the targeted genes were involved in the $\mathrm{NF \kappa B}$ signaling pathway. These data suggest a potential causal relationship between rhinovirus infection-induced programing of airway cells (i.e., epigenetic changes via microRNAs inducing NFKB signaling mediators), and the development of asthma in young children. However, it is also possible that the altered airway microRNA profiles and enhanced NFKB signaling in the setting of rhinovirus infection may simply be a marker of an individual who is prone to develop childhood asthma. In addition, the underlying mechanisms linking severe virus infection to incident asthma may differ among different asthma phenotypes (e.g., atopic vs. nonatopic asthma) (2). Notwithstanding this complexity, the identification of distinct airway microRNA profiles and enhanced NFkB signaling pathway in infants with rhinovirus is an important advance.

Several potential limitations of our study should be taken into account. First, bronchiolitis involves inflammation of the lower airway from which specimen sampling is ethically and technically challenging in infants. Although our study was based on the nasal airway samples, the literature has reported strong correlations between upper and lower airway virology (44), gene expression $(19,20)$, and inflammatory mediators (21). Therefore, the microRNA and inflammatory profiles in the nasal airway are likely indicative of those in the lower airways. Second, as our samples were not cell-based, we did not measure NFKB activity per se. Nevertheless, we measured the gene expression for the components of NFkB signaling pathway, as well as the cytokines 


\section{Articles | Hasegawa et al.}

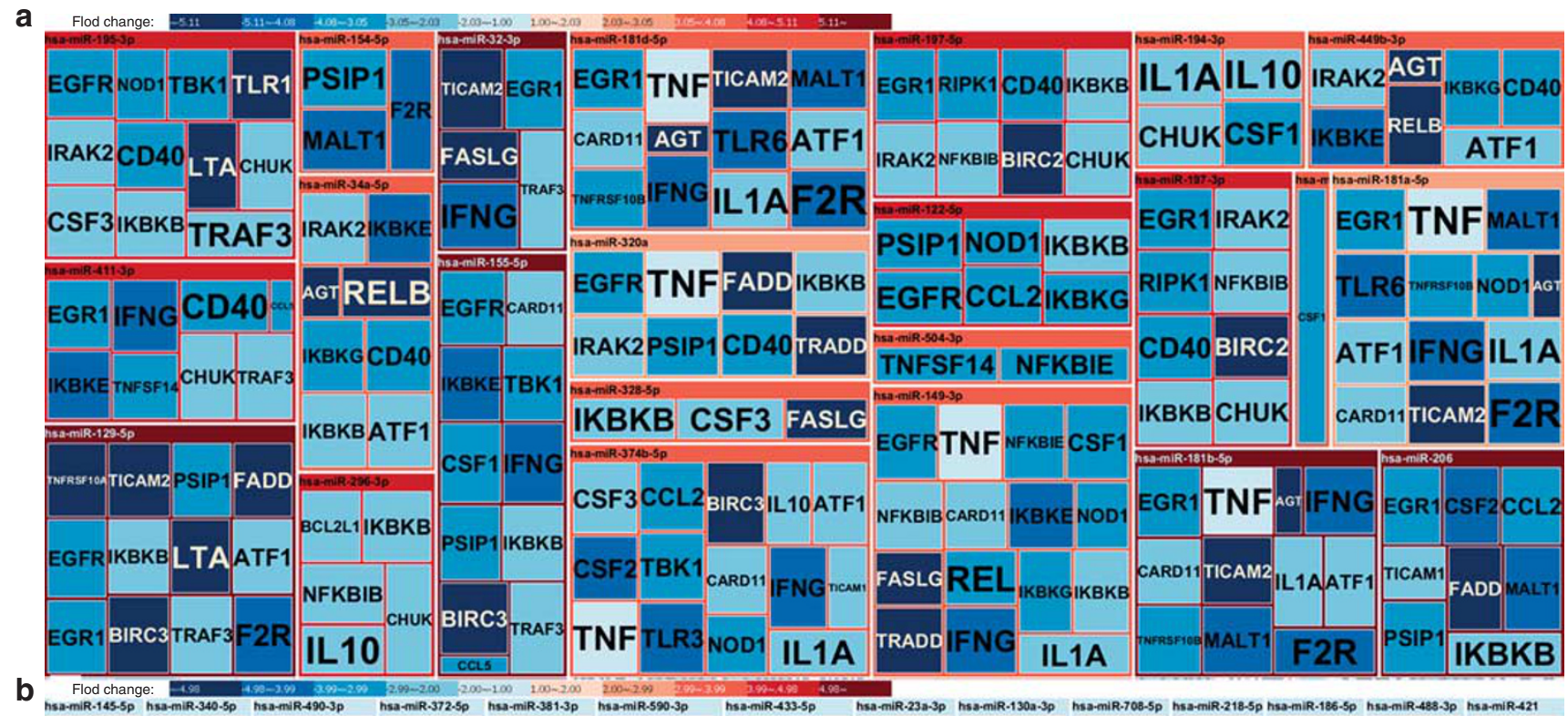

BCL3 FOS ICAM1 RELA FOS FOS FOS BCL3 ICAM1 RELA BCL3 RELA RELA RELA $B C L 3$ FOS FOS RELA ICAM1 RELA RELA RELA FOS RELAICAM1 ICAM1 BCL3 RELA RELA RELA FOS ICAM1 RELA FOS FOS

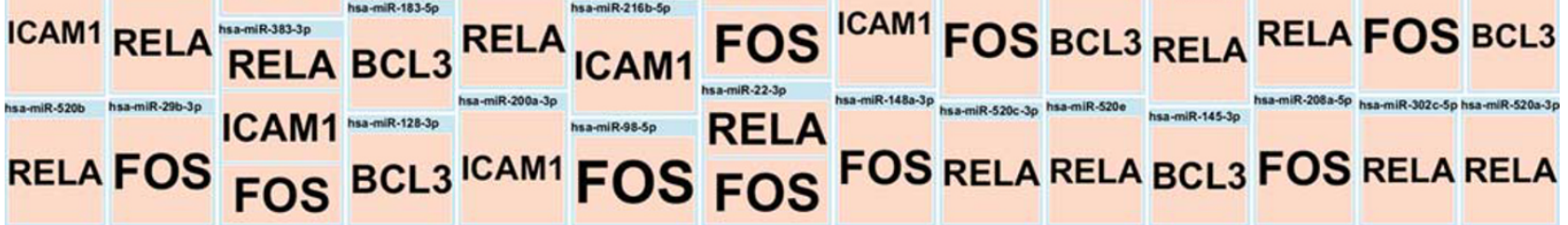

Figure 4. Integrated analysis of microRNA and mRNA expressions of NFkB signaling pathway in nasal airway of infants with bronchiolitis. Treemap of predicted microRNA-target (mRNA) interaction was depicted by the use of miRTarVis, which identifies microRNA-mRNA pairs with an expression value. Normalized, background-subtracted microRNA-mRNA expression profile data were imported into miRTarVis (rhinovirus infection compared to RSV infection). The color gradient indicates the magnitude of the fold change in microRNA and mRNA expression (red, upregulation; blue, downregulation). The size (area) of each box represents the frequency of that finding. The shape is designed to automatically fit into the overall structure of the figure. (a) Pairs of upregulated microRNAs (in red) and downregulated mRNA (in blue). Notably, infants with rhinovirus bronchiolitis had upregulation of multiple microRNAs (e.g., hsa-miR-149-3p, hsa-miR-197-3p, hsa-miR-197-5p, and hsa-miR-296-3p) targeting NFKBIB, thereby predicting the downregulation of $N F K B I B$, a gene encoding inhibitor KB family. (b) Pairs of downregulated microRNAs (in blue) and upregulated mRNA (in red). Notably, infants with rhinovirus bronchiolitis had downregulation of multiple microRNAs targeting RELA, thereby predicting the upregulation of $R E L A$, the gene encoding RelA (one of the proteins in the NFKB family).

(e.g., IL-10, IL-13) for inflammatory mediators induced by NFkB. Third, the observed differences in microRNA profiles may be attributable to potential differences in cellular profiles by virus. However, we removed cellular RNAs by filtering cells. Fourth, the present study design precluded us from examining the relation between longitudinal patterns of the microRNAmediated airway immune modulation and respiratory health in children (e.g., development of asthma). To address this question, the study population is currently being followed to 6 years of age with nasal airway specimen sampling at multiple time points.
Fifth, we did not have the data of a "control" group, such as healthy infants without respiratory virus infection. Yet, the study objective was not to evaluate the role of microRNA on the development of bronchiolitis (yes/no), but to determine the virus-specific pathobiology involving airway microRNAs within infants with bronchiolitis (rhinovirus vs. RSV). Sixth, while the current study demonstrated the findings to be consistent by examining both predicted and measured gene expression, external validation would be necessary to confirm these observations. Lastly, we must generalize our findings cautiously 
beyond infants who had severe bronchiolitis. Nonetheless, our data remain highly relevant for 130,000 children hospitalized for bronchiolitis in the US each year (1).

\section{CONCLUSIONS}

In this multicenter cohort study of infants hospitalized with bronchiolitis, we found that nasal airway microRNA profiles differ between the two most common viruses causing bronchiolitis, rhinovirus, and RSV. Our data also demonstrated that infants with rhinovirus infection had an altered microRNA profile that is predicted to enhance the NFKB signaling pathway. Conversely, infants with RSV infection had a microRNA profile that is predicted to have a downregulated $\mathrm{NFKB}$ signaling pathway. These findings were validated by the observation that microRNA signature in rhinovirus infection is associated with measured upregulation of $\mathrm{NFkB}$ genes and downregulation of IкB family genes. In addition, infants with rhinovirus had higher levels of NFkB-induced type-2 cytokines (IL-10 and IL-13) compared to those with RSV infection. In conjunction with prior studies, our data suggest a potential mechanism linking rhinovirus infection and bronchiolitis-related chronic morbidities-i.e., rhinovirus infection-induced programing of airway cells, via epigenetic changes involving microRNAs, induces NFKB signaling mediators and unique immune response profiles. Our data should facilitate further mechanistic investigations to disentangle the complex web of viral pathogens, microRNA regulation, and host immune responses in the airway of young children with bronchiolitis.

\section{SUPPLEMENTARY MATERIAL}

Supplementary material is linked to the online version of the paper at http://www.nature.com/pr

\section{ACKNOWLEDGMENTS}

We thank Ashley Sullivan, Courtney Tierney, and Janice Espinola at the EMNet Coordinating Center (Massachusetts General Hospital, Boston, MA), and all of the site investigators and study staff for their valuable contributions to the MARC-35 study. We also thank Alkis Togias at the National Institutes of Health (Bethesda, MD) for helpful comments about the study results. Lastly, we thank the participating families for making all of this possible.

\section{STATEMENT OF FINANCIAL SUPPORT}

This study was supported by the grants UG3 OD-023253, U01 Al-087881, R01 Al-114552, R01 Al-108588, R01 Al-127507, R21 HL-129909, and K12 HL-119994 from the National Institutes of Health (Bethesda, MD).

\section{DISCLAIMER}

The content of this manuscript is solely the responsibility of the authors and does not necessarily represent the official views of the National Institutes of Health.

Disclosure: J.M.M. has provided bronchiolitis-related consultation for Regeneron. P.A.P. received research grants from Gilead, Janssen Vaccines and Prevention, Novavax, and Regeneron, and provided bronchiolitisrelated consultation for Ablynx, LFB, Medlmmune, Novavax, and Regeneron. All the remaining authors declare no conflict of interest.

\section{REFERENCES}

1. Hasegawa K, Tsugawa Y, Brown DF, Mansbach JM, Camargo CA Jr. Trends in bronchiolitis hospitalizations in the United States, 2000-2009. Pediatrics 2013;132:28-36.
2. Hasegawa K, Mansbach JM, Camargo CA Jr. Infectious pathogens and bronchiolitis outcomes. Expert Rev Anti Infect Ther 2014;12:817-28.

3. Mejias A, Dimo B, Suarez NM, et al. Whole blood gene expression profiles to assess pathogenesis and disease severity in infants with respiratory syncytial virus infection. PLoS Med 2013;10:e1001549.

4. Hasegawa K, Dumas O, Hartert TV, Camargo CA Jr. Advancing our understanding of infant bronchiolitis through phenotyping and endotyping: clinical and molecular approaches. Exp Rev Respir Med 2016;10:891-9.

5. Foster PS, Plank M, Collison A, et al. The emerging role of microRNAs in regulating immune and inflammatory responses in the lung. Immunol Rev 2013;253:198-215.

6. Friedman RC, Farh KK, Burge CB, Bartel DP. Most mammalian mRNAs are conserved targets of microRNAs. Genome Res 2009;19:92-105.

7. Gutierrez MJ, Gomez JL, Perez GF, et al. Airway secretory microRNAome Changes during Rhinovirus Infection in Early Childhood. PLoS One 2016;11:e0162244.

8. Ilarraza R, Wu Y, Skappak CD, Ajamian F, Proud D, Adamko DJ. Rhinovirus has the unique ability to directly activate human $\mathrm{T}$ cells in vitro. J Allergy Clin Immunol 2013;131:395-404.

9. Papi A, Contoli M, Adcock IM, et al. Rhinovirus infection causes steroid resistance in airway epithelium through nuclear factor kappaB and c-Jun N-terminal kinase activation. J Allergy Clin Immunol 2013;132:1075-85 e6.

10. Bartlett NW, Slater L, Glanville N, et al. Defining critical roles for NFkappaB p65 and type I interferon in innate immunity to rhinovirus. EMBO Mol Med 2012;4:1244-60.

11. Hewson CA, Haas JJ, Bartlett NW, et al. Rhinovirus induces MUC5AC in a human infection model and in vitro via NF-kappaB and EGFR pathways. Eur Respir J 2010;36:1425-35.

12. Girkin J, Hatchwell L, Foster P, et al. CCL7 and IRF-7 mediate hallmark inflammatory and IFN responses following rhinovirus $1 \mathrm{~B}$ infection. J Immunol 2015;194:4924-30.

13. Hasegawa K, Mansbach JM, Ajami NJ, et al. Association of nasopharyngeal microbiota profiles with bronchiolitis severity in infants hospitalized for bronchiolitis. Eur Respir J 2016;48:1329-39.

14. Stewart CJ, Mansbach JM, Wong MC, et al. Associations of nasopharyngeal metabolome and microbiome with severity among infants with bronchiolitis: a multi-omic analysis. Am J Respir Crit Care Med 2017;196:882-91.

15. Hasegawa K, Mansbach JM, Ajami NJ, et al. Serum cathelicidin, nasopharyngeal microbiota, and disease severity in infants hospitalized with bronchiolitis. J Allergy Clin Immunol 2016;139:1383-86.e6.

16. Mansbach JM, Hasegawa K, Henke DM, et al. Respiratory syncytial virus and rhinovirus severe bronchiolitis are associated with distinct nasopharyngeal microbiota. J Allergy Clin Immunol 2016;137:1909-13 e4.

17. Hasegawa K, Mansbach JM, Ajami NJ, et al. The relationship between nasopharyngeal CCL5 and microbiota on disease severity among infants with bronchiolitis. Allergy 2017;72:1796-800.

18. Ralston SL, Lieberthal AS, Meissner HC, et al. American Academy of Pediatrics. Clinical practice guideline: the diagnosis, management, and prevention of bronchiolitis. Pediatrics 2014;134:e1474-502.

19. Poole A, Urbanek C, Eng C, et al. Dissecting childhood asthma with nasal transcriptomics distinguishes subphenotypes of disease. J Allergy Clin Immunol 2014;133:670-8e12.

20. Sridhar S, Schembri F, Zeskind J, et al. Smoking-induced gene expression changes in the bronchial airway are reflected in nasal and buccal epithelium. BMC Genomics 2008;9:259.

21. McDougall CM, Blaylock MG, Douglas JG, Brooker RJ, Helms PJ, Walsh GM. Nasal epithelial cells as surrogates for bronchial epithelial cells in airway inflammation studies. Am J Respir Cell Mol Biol 2008;39:560-8.

22. Lambert $\mathrm{SB}$, Ware RS, Cook $\mathrm{AL}$, et al. Observational research in childhood infectious diseases (ORChID): a dynamic birth cohort study. PLoS One 2013;8:e63871.

23. Damsker JM, Dillingham BC, Rose MC, et al. VBP15, a glucocorticoid analogue, is effective at reducing allergic lung inflammation in mice. PLoS One 2013;8:e63871. 


\section{Articles | Hasegawa et al.}

24. Kerr DM, Harhen B, Okine BN, Egan LJ, Finn DP, Roche M. The monoacylglycerol lipase inhibitor JZL184 attenuates LPS-induced increases in cytokine expression in the rat frontal cortex and plasma: differential mechanisms of action. Br J Pharmacol 2013;169:808-19.

25. Yagi O, Aoshiba K, Nagai A. Activation of nuclear factor-kappaB in airway epithelial cells in patients with chronic obstructive pulmonary disease. Respiration 2006;73:610-6.

26. Read MA, Whitley MZ, Williams AJ, Collins T. NF-kappa B and I kappa B alpha: an inducible regulatory system in endothelial activation. J Exp Med 1994;179:503-12.

27. Jung D, Kim B, Freishtat RJ, Giri M, Hoffman E, Seo J. miRTarVis: an interactive visual analysis tool for microRNA-mRNA expression profile data. BMC Proc. 2015;9(Suppl 6 Proceedings of the 5th Symposium on Biological Data):S2.

28. Tu YC, Huang DY, Shiah SG, Wang JS, Lin WW. Regulation of c-Fos gene expression by NF-kappaB: a p65 homodimer binding site in mouse embryonic fibroblasts but not human HEK293 cells. PLoS ONE 2013;8:e84062.

29. Fujioka S, Niu J, Schmidt C, et al. NF-kappaB and AP-1 connection: mechanism of NF-kappaB-dependent regulation of AP-1 activity. Mol Cell Biol 2004;24:7806-19.

30. Hinz M, Lemke P, Anagnostopoulos I, et al. Nuclear factor kappaBdependent gene expression profiling of Hodgkin's disease tumor cells, pathogenetic significance, and link to constitutive signal transducer and activator of transcription 5a activity. J Exp Med 2002;196: 605-17.

31. Cao S, Zhang X, Edwards JP, Mosser DM. NF-kappaB1 (p50) homodimers differentially regulate pro- and anti-inflammatory cytokines in macrophages. J Biol Chem 2006;281:26041-50.

32. Jartti T, Paul-Anttila M, Lehtinen P, et al. Systemic T-helper and T-regulatory cell type cytokine responses in rhinovirus vs. respiratory syncytial virus induced early wheezing: an observational study. Respir Res 2009;10:85.

33. Thornburg NJ, Hayward SL, Crowe JE Jr. Respiratory syncytial virus regulates human microRNAs by using mechanisms involving beta interferon and NF-kappaB. MBio 2012;3: pii: e00220-12.
34. Inchley CS, Sonerud T, Fjaerli HO, Nakstad B. Nasal mucosal microRNA expression in children with respiratory syncytial virus infection. BMC Infect Dis 2015;15:150.

35. Zech A, Ayata CK, Pankratz F, et al. MicroRNA-155 modulates P2R signaling and Th2 priming of dendritic cells during allergic airway inflammation in mice. Allergy 2015;70:1121-9.

36. Okoye IS, Czieso S, Ktistaki E, et al. Transcriptomics identified a critical role for Th2 cell-intrinsic miR-155 in mediating allergy and antihelminth immunity. Proc Natl Acad Sci USA 2014;111:E3081-90.

37. Martinez-Nunez RT, Louafi F, Sanchez-Elsner T. The interleukin 13 (IL-13) pathway in human macrophages is modulated by microRNA-155 via direct targeting of interleukin 13 receptor alpha1 (IL13Ralpha1). J Biol Chem. 2011;286:1786-94.

38. Malmhall C, Alawieh S, Lu Y, et al. MicroRNA-155 is essential for T(H)2mediated allergen-induced eosinophilic inflammation in the lung. J Allergy Clin Immunol 2014;133:1429-38,38 e1-7.

39. Tully JE, Hoffman SM, Lahue KG, et al. Epithelial NF-kappaB orchestrates house dust mite-induced airway inflammation, hyperresponsiveness, and fibrotic remodeling. J Immunol 2013;191:5811-21.

40. Ather JL, Hodgkins SR, Janssen-Heininger YM, Poynter ME. Airway epithelial NF-kappaB activation promotes allergic sensitization to an innocuous inhaled antigen. Am J Respir Cell Mol Biol 2011;44:631-8.

41. Hart LA, Krishnan VL, Adcock IM, Barnes PJ, Chung KF. Activation and localization of transcription factor, nuclear factor-kappaB, in asthma. Am J Respir Crit Care Med 1998;158 (5 Pt 1): 1585-92.

42. Gagliardo R, Chanez P, Mathieu M, et al. Persistent activation of nuclear factor-kappaB signaling pathway in severe uncontrolled asthma. Am J Respir Crit Care Med. 2003;168:1190-8.

43. Panganiban RP, Wang Y, Howrylak J, et al. Circulating microRNAs as biomarkers in patients with allergic rhinitis and asthma. J Allergy Clin Immunol 2016;137:1423-32.

44. Mosser AG, Vrtis R, Burchell L, et al. Quantitative and qualitative analysis of rhinovirus infection in bronchial tissues. Am J Respir Crit Care Med. 2005;171:645-51. 